\title{
The Solution of Single Index Portfolio Selection Model with Transaction Cost by Genetic Algorithm
}

\author{
Xinling Feng \\ Jiangsu University of technology \\ Jiangsu, China \\ E-mail: lucky8429@sina.com \\ Cuiqing Zhu \\ Jiangsu University of technology \\ Jiangsu, China
}

\author{
Sheng $\mathrm{He}$ \\ Jiangsu University of technology \\ Jiangsu, China \\ E-mail: hs@jsut.edu.cn \\ E-mail: zcq@jsut.edu.cn
}

\begin{abstract}
The key to the portfolio optimization problem is to achieve effective income risk allocation. Using single index model brings forward portfolio model with transaction costs and reduces the calculation of the investment portfolio covariance matrix. At the same time, use genetic algorithm to solve objective function and combine actual data to make simulation experiment. It is turned out that genetic algorithm can be used to better solve the model and the results reflect the rationality of the model.
\end{abstract}

Keywords-Investment Portfolio; Transaction Cost; Single Index Model; Genetic Algorithm

\section{INTRODUCTION}

In 1952, the famous American economist Markowitz published Portfolio Selection [1] and put forward the thought and methods to determine the minimum variance portfolio collection, which laid the cornerstone of the portfolio investment theory and marked the beginning of modern investment portfolio theory. In Markowitz's model, the optimal portfolio selection is transformed into optimization to solve the problem, that is to say, under a certain level, to minimize the risk; or under a certain level of risk, maximize the income. That is, carry out the portfolio selection through quadratic programming model. However, when there are many variables, for the Markowitz model, the amount of calculation of covariance matrix is big and the actual operation is difficult to achieve. In view of the above shortcomings of Markowitz model, scholars have improved the model. Sharpe put forward the Single Index Model. [2] Compared with Markowitz model, although the precision of the calculation of single index model is reduced, the income of its hypothetical assets is only related to the market overall average yield. And the relationship between the securities is connected by the market, which greatly simplifies the calculation of the model. [3] Besides, Markowitz model is based on strong hypothesis, without thinking problems such as not allowing short selling and short purchase, trade costs and minimum trading units, etc. There are problems in practical operation. In recent years, many scholars do some researches on this [4] [5] [6], but they still cannot get rid of the problem of the too complex calculation of covariance matrix. The article will use the single index model of Sharpe and consider the problems such as short selling and short purchase, trade costs and minimum trading results, establish objective function and use genetic algorithm to solve the results.

\section{EST ABLISHING OBJECTIVE FUNCTION}

Assume that there are $\mathrm{n}$ kinds of securities in the investment portfolio, ${ }^{r}$ as the yield rate of the number $i$ security, ${ }^{R_{i}}$ as the yield rate, $E\left(r_{i}\right)$ as the expectation, $x_{i}$ as the investment proportion of the number $i$ security, then the expected yield rate of the security portfolio is $R_{p}=\sum_{i=1}^{n} x_{i} R_{i} \quad \sigma_{p}^{2}=\sum_{i=1}^{n} \sum_{j=1}^{n} x_{i} x_{j} \sigma_{i j}$ ( $\sigma_{i j}$ is the covariance of security $i$ and $j$ ). The risk is the minimum. At the same time, there are contradictions between investment portfolios with the largest investment income. Researchers cannot have both of them. For this purpose, the Markowitz model is expressed as the following optimal problem:

$$
\left\{\begin{array}{c}
\max (1-\omega) \sum_{i=1}^{n} x_{i} R_{i}-\omega \sum_{i=1}^{n} \sum_{j=1}^{n} x_{i} x_{j} \sigma_{i j} \\
\text { st. } \quad \sum_{i=1}^{n} x_{i}=1
\end{array}\right.
$$

$\omega$ is the risk reference coefficient. $\omega=0$ shows the investors only pursue incomes and don't care. $\omega=1$ shows the investors don't care about the income and they only hope to minimize the investment risk. $\omega \in[0,1]$ means the investors' degree of preference for risk. 
As stated in the preface, when Markowitz model is applied to practical work, the portfolio needs to be operated by a set of advanced and sophisticated computer programs and the calculated amount is too large. While the single index model found by Sharpe only greatly simplifies the calculating process of portfolio selection. The single index model assumes that the assets income is only related to the market overall revenue, thus educe $r_{i}=\alpha_{i}+\beta_{i} r_{m}+e_{i} \alpha_{i}$ is the expectation of the part which is independent of the market gains in income. $\beta_{i}$ is the sensitive coefficient of relative market of the number $i_{\text {security. }} r_{m}$ is the yield rate of market index. $e_{i}$ is the random error term. $e_{i}$ and $r_{m}$ are the random variable, and $\sigma_{e i}$ and $\sigma_{m}$ as their standard deviation respectively. Assume that $E\left(e_{i}\right)=0, D\left(e_{i}\right)=\sigma_{e i}$, and the above formula also requires to meet conditions: $\mathrm{E}\left(e_{i}, e_{j}\right)=0(i \neq j)$ and $E\left[e_{i}\left(\mathrm{r}_{m}-R_{m}\right)\right]=0$. Educe the expectation of yield rate is: $R_{i}=\alpha_{i}+\beta_{i} R_{m}$. The variance of securities return rate is $\sigma_{i}^{2}=\beta_{i}^{2} \sigma_{m}^{2}+\sigma_{e i}^{2}$. The covariance between security $i$ and $j$ is $\sigma_{i j}=\beta_{i} \beta_{j} \sigma_{m}^{2}$. Then the yield rate expectation of the investment

$$
\begin{aligned}
& R_{p}=\sum_{i=1}^{n} x_{i} R_{i}=\sum_{i=1}^{n} x_{i} \alpha_{i}+\sum_{i=1}^{n} x_{i} \beta_{i} R_{m} \\
& { }_{\text {Make }} \beta_{p}=\sum_{i=1}^{n} x_{i} \beta_{i} \quad \alpha_{p}=\sum_{i=1}^{n} x_{i} \alpha_{i}, \text { then } \\
& R_{p}=\alpha_{p}+\beta_{p} R_{m}
\end{aligned}
$$

The variance of the investment portfolio

$$
\begin{aligned}
\sigma_{p}^{2} & =\sum_{i=1}^{n} x_{i}^{2} \sigma_{i}^{2}+\sum_{i=1}^{n} \sum_{\substack{j=1 \\
i \neq j}}^{n} x_{i} x_{j} \sigma_{i j} \\
& =\sum_{i=1}^{n} x_{i}^{2} \beta_{i}^{2} \sigma_{m}^{2}+\sum_{i=1}^{n} \sum_{\substack{j=1 \\
i \neq j}}^{n} x_{i} x_{j} \beta_{i} \beta_{j} \sigma_{m}^{2}+\sum_{i=1}^{n} x_{i}^{2} \sigma_{e i}^{2} \\
& =\left(\sum_{i=1}^{n} x_{i} \beta_{i}\right)\left(\sum_{j=1}^{n} x_{j} \beta_{j}\right) \sigma_{m}^{2}+\sum_{i=1}^{n} x_{i}^{2} \sigma_{e i}^{2} \\
& =\beta_{p}^{2} \sigma_{m}^{2}+\sum_{i=1}^{n} x_{i}^{2} \sigma_{e i}^{2}
\end{aligned}
$$

From this, researchers know that the investment portfolio variance consists of system risk $\beta_{p}^{2} \sigma_{m}^{2}$ and non$\sum_{i=1}^{n} x_{i}^{2} \sigma_{e i}^{2}$ controlled by investors, they adjust the non-system risk by
Markowitz model assumes the trade share can be divided infinitely. However, in real trade, there are usually problems of minimum trading unit. For example, domestic follow that when buying stocks, the minimum unit is one hand (100 shares). Under this circumstance, the efficient frontier of investment portfolio is not consisted of a continuous curve but a series of discontinuous points. If the variables in the model are still expressed by investment proportion, then it may cause bigger error in the process of calculation, which may contradict with $\sum_{i=1}^{n} x_{i}=1$ that $C 、 C_{0} 、 C_{1}$ are investment amount, the minimum amount of investment and the maximum amount, $p_{i}$ as the price of the number $i$ security and $a$ as the amount contained by one hand security, and $\mathrm{Y}=\left(y_{1}, y_{2}, \cdots y_{n}\right)$ is an investment portfolio. Among them, $y_{i}$ is the hand number of the number $i$ security. Under the limits of China securities market of not allowing short selling and short purchase, $y_{i}$ is a nonnegative integer. Therefore, the investment amount is $C=a \sum_{i=1}^{n} y_{i} p_{i}$ and the investment proportion of number $i$ security is $\eta_{i}=\frac{a y_{i} p_{i}}{C}$ Markowitz didn't consider the transaction cost. However, in actual transaction operation, national tax authorities, exchanges and security companies charge transaction costs from investor according to certain proportion, such as trading commission, stamp tax and transfer fee, etc. Practice has proved that compared with trading volume, although the amount of transaction cost is small, it neglects that transaction cost always leads to invalid stock portfolio. Assume $k_{i}$ is the fixed proportion of transaction costs account for trading volume, and $C_{i}$ is the transaction costs needed by number $i$ security. The original investment portfolio of the investors is $\left(y_{1}^{0}, y_{2}^{0} \cdots y_{n}^{0}\right)$ and the new investment portfolio is $\left(y_{1}, y_{2} \cdots y_{n}\right)$, and then $C_{i}=k_{i}\left|\eta_{i}-\eta_{i}^{0}\right|$. And the total transaction cost $\sum_{i=1}^{n} C_{i}=\sum_{i=1}^{n} k_{i}\left|\eta_{i}-\eta_{i}^{0}\right|$ function of the investors is:

$$
\sum_{i=1}^{n} \eta_{i} \alpha_{i}+\sum_{i=1}^{n} \eta_{i} \beta_{i} R_{m}-\sum_{i=1}^{n} k_{i}\left|\eta_{i}-\eta_{i}^{0}\right|
$$

The totalvariance function is:

$$
\left(\sum_{i=1}^{n} \eta_{i} \beta_{i}\right)^{2} \sigma_{m}^{2}+\sum_{i=1}^{n} \eta_{i}^{2} \sigma_{e i}^{2}
$$


Further assume that $h_{i}$ is the biggest hand number [8] permitted to be invested in number $i$ security $\mathrm{S}$, finally,

$$
\begin{aligned}
& \max \quad f(Y)=(1-\omega)\left(\sum_{i=1}^{n} \eta_{i} \alpha_{i}+\sum_{i=1}^{n} \eta_{i} \beta_{i} R_{m}-\sum_{i=1}^{n} k_{i} \mid\right. \\
& \text { s.t }\left\{\begin{array}{l}
C_{0} \leq C=a \sum_{i=1}^{n} y_{i} p_{i} \leq C_{1} \\
0 \leq y_{i} \leq h_{i} \text { 且 } y_{i} \in N
\end{array}\right.
\end{aligned}
$$

Among them, the first constraint is the limit for $\mathrm{C}$. It is generally demand $\left(C_{1}-C_{0}\right) / C_{0}$ is a small positive number. It ensures almost all the funds are put into the investment. This condition is corresponding to the constraint $\sum_{i=1}^{n} x_{i}=1$ in Markowitz model. The objective function is nonlinear integer programming model and it is NP problem. Traditional methods are difficult to find global optimal solution.

\section{MODEL SOLVING}

Genetic Algorithm is a calculation model that simulates the natural selection of Darwin's biological theory of evolution and biologic evolution process of genetic mechanism, and a kind of method to search the optimal solution through simulating natural evolution process. It has the characteristics of highly parallel, random and adaptive search and has the ability to search the global optimum in theory. [7] However, when actually seeking the optimal value, it uses fixed selective probability and crossover probability to make traditional genetic algorithm cause premature convergence at the early evolution. Thus in the middle and later evolutionary process, it lacks individual diversity and reduces the algorithm search ability and finally converges to local optimal solution. [9] Therefore, researchers use adaptive genetic algorithm to solve the objective function. And the specific algorith ms of the adaptive genetic algorithm [10] suggested is as follows:

\section{A. Encoding}

A security is corresponding to a gene position. A

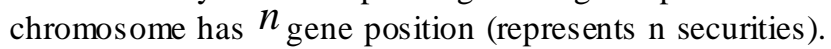
Using integer coding, the value of the gene represents the hand number of this portfolio investment.

\begin{tabular}{|l|l|l|l|l|l|}
\hline$x_{1}$ & $x_{2}$ & $x_{3}$ & $x_{4}$ & $\cdots$ & $x_{n}$ \\
\hline
\end{tabular}

\section{B. Fitness Function}

Because choice mechanis $m$ is roulette wheel selection method, the fitness function is defined as $e^{f(Y)}$.It ensures the fitness function is greater than zero.

\section{Generate the Initial Group}

The integer $N$ generated by the initial group is the number of chromosomes and use integer coding. The improve Markowitz model, the objective function can be expressed as: 
Step 3: Generate random number $r \in\left(0, p p_{N}\right)$.If $p p_{j-1}<r<p p_{j}$, then select the number $j$ chromosome $(1 \leq j \leq N)$.

Step 4: Repeat Step 2, Step 3 for $N$ times, and then researchers can get $N$ replicated chromosome $V^{\prime}(k)=\left(V_{1}^{\prime}(k), V_{2}^{\prime}(k), \cdots V_{N}^{\prime}(k)\right)$

\section{E. Improved Crossover and Mutation Operation}

(1) Autoregulative crossover probability and mutation probability

Cross over and mutation operator play a decisive role in the convergence of genetic algorithm. In order to avoid the occurrence of divergence and getting into local optimum, keep good individual and speed up the optimization, researchers improve crossover and mutation operation. The measure to checkout the genetic algorithm convergence uses the fitness function $e_{\max }^{f}$ of optimal individual and average fitness function $e_{\min }^{f}$ of the group to automatically adjust the crossover probability $p_{c}$ and mutation probability $p_{m}$ [7] [10]:

$$
\begin{aligned}
& p_{c}=\left\{\begin{array}{l}
\sin \left(\frac{\pi}{2} \frac{e_{\max }^{f}-e^{f^{\prime}}}{e_{\max }^{f}-e_{\text {ave }}^{f}}\right), e^{f^{\prime}}>e_{\text {ave }}^{f} ; \\
1, \quad, e^{f^{\prime}} \leq e_{\text {ave }}^{f} ;
\end{array}\right. \\
& p_{m}=\left\{\begin{array}{c}
0.5 \sin \left(\frac{\pi}{2} \frac{e_{\max }^{f}-e^{f^{\prime}}}{e_{\max }^{f}-e_{\text {ave }}^{f}}\right), e^{f^{\prime}}>e_{\text {ave }}^{f} \\
0.5, \quad, e^{f^{\prime}} \leq e_{\text {ave }}^{f} ;
\end{array}\right.
\end{aligned}
$$

(2) Crossoveroperation

Regard $p_{c}$ as mutation probability, use crossover operation, and reallocate investment portfolio. This must be processed in the same period of parent individuals. Randomly generate two cross points $k$ and $j(1 \leq k<j \leq n)$. New individual generated after the operation cannot always meet the first constraint condition of the objective function, but it still must meet the second constraint condition. Therefore, new individuals need feasible treatment.

(3) Mutation operation

Regard $p_{m}$ as mutation probability, and the mutation operation is equal to make a fine tuning for distribution of the investment portfolio. Randomly generate mutant gene $k \quad(1 \leq k \leq n)$, then carry out the following operation:

$$
y_{k}=\operatorname{rand} \operatorname{int}\left(0, h_{k}\right)
$$

New individual generated after the mutation operation cannot always meet the first constraint condition of the objective function, but it still must meet the second constraint condition. Therefore, new individuals need feasible treatment.

\section{F. After Selection, Crossover And Mutation, Generate A New Generation of Chromosomes and Record the Best Chromosomes.}

Give the iteration $G$ and operate $G$ times. Finally find out the optimal solution.

\section{ANALYSIS OF EXAMPLES}

Use matlab software to write the procedure. [11] Assume $a=100, C_{0}=200, C_{1}=200.50$. According to the current situation of China, the transaction cost of security calculate by $k_{i}=0.005$, assume $h_{i}=3000$ Select five kinds of stocks of Shanghai Pudong Development Bank, Port of Nanjing, Qianjiang Biochemical, Suning Appliance and Shandong Gold as investment portfolio and the initial investment is zero hand. Download the weekly closing price data during January 11 , 2008 and February 10, 2012 from the Great Wisdom, use matlab software regression and get $\alpha_{i}, \beta_{i}, R_{m}, \sigma_{m}^{2}, \sigma_{e i}^{2}$, as Table 1 below.

TABLE I. ThE VALUES OF STOCKS $\alpha_{i}, \beta_{i}, \sigma_{e i}^{2}$

\begin{tabular}{llll}
\hline Stock Name & $\alpha_{i}$ & $\beta_{i}$ & $\sigma_{e i}^{2}$ \\
\hline $\begin{array}{l}\text { Shanghai Pudong } \\
\text { Development Bank }\end{array}$ & 0.003 & 1.239 & 0.0444 \\
Nanjing Port & 0.002 & 1.176 & 0.04968 \\
Qianjiang Biochemical & 0.007 & 1.220 & 0.06765 \\
Suning Electric Appliance & 0.001 & 0.754 & 0.0461337 \\
Shandong Gold & 0.008 & 1.028 & 0.0681058 \\
\hline
\end{tabular}

Substitute the data in table 1 into the objective function. Calculate with computer, researchers can get the optimal combination under different investment risk preference, as Table 2. 
TABLE II. OPTIMAL COMBINATION UNDER DIFFERENT NVESTMENT RISK PREFERENCE

\begin{tabular}{|c|c|c|c|c|c|c|c|c|}
\hline \multirow[t]{2}{*}{$\omega$} & \multicolumn{5}{|l|}{ Lots number } & \multirow{2}{*}{$\begin{array}{l}\text { Total market value } \\
\text { (yuan) }\end{array}$} & \multirow{2}{*}{$\begin{array}{l}\% \\
\text { Income } \%\end{array}$} & \multirow{2}{*}{$\begin{array}{l}\% \\
\text { Risk\% }\end{array}$} \\
\hline & $\begin{array}{c}\text { Shanghai } \\
\text { Pudong } \\
\text { Development } \\
\text { Bank } \\
\end{array}$ & $\begin{array}{c}\text { Nanjing } \\
\text { Port }\end{array}$ & $\begin{array}{c}\text { Qianjiang } \\
\text { Biochemical }\end{array}$ & $\begin{array}{c}\text { Suning Electric } \\
\text { Appliance }\end{array}$ & $\begin{array}{l}\text { Shandong } \\
\text { Gold }\end{array}$ & & & \\
\hline 0 & 0 & 0 & 0 & 0 & 566 & 2004206 & 0.42 & 6.99 \\
\hline 0.1 & 244 & 0 & 1038 & 191 & 257 & 2002529 & 0.25 & 2.51 \\
\hline 0.2 & 378 & 460 & 710 & 379 & 163 & 2001961 & 0.10 & 1.50 \\
\hline 0.3 & 430 & 587 & 603 & 444 & 133 & 2002322 & 0.05 & 1.34 \\
\hline 0.4 & 452 & 661 & 553 & 480 & 116 & 2003009 & 0.02 & 1.29 \\
\hline 0.5 & 468 & 683 & 523 & 502 & 108 & 2001695 & 0.007 & 1.28 \\
\hline 0.6 & 479 & 707 & 504 & 517 & 102 & 2004945 & -0.002 & 1.27 \\
\hline 0.7 & 485 & 725 & 488 & 526 & 97 & 2000316 & -0.01 & 1.27 \\
\hline 0.8 & 492 & 739 & 478 & 534 & 94 & 2004578 & -0.0148 & 1.26 \\
\hline 0.9 & 496 & 750 & 469 & 540 & 91 & 2003226 & -0.0193 & 1.26 \\
\hline 1.0 & 500 & 757 & 462 & 545 & 89 & 2003619 & -0.0225 & 1.26 \\
\hline
\end{tabular}

\section{CONCLUSIONS}

This article uses the single index model of Sharpe, which greatly simplifies the counting process of optimal investment portfolio. Besides, it uses adaptive genetic algorithm, which solves the NP problem of nonlinear integer programming. In securities investment decision, it has more practicability. Using genetic algorithm solves this model and uses matlab software to compose the program. Through instance simulation, the feasibility of this algorithm has been verified and it has achieved satisfactory results.

\section{REFERENCES}

[1] H Markowitz. Portfolio selection[J]. Finance. 1952(3):77-91

[2] W F Sharpe. A Simplified Model for Portfolio Analysis[J]. Managemint Science. 1963, 10(2):277-293

[3] Jin Hanjun, Wang Hongfeng. Model Analysis on Stock Portfolio Investment Optimization Problem Based on Genet ic Algorithm [J] Journal of Huazhong Normal University, 2004,12,38(4):427-429

[4] Bai Xianchun. Genetic Algorithm of Portfolio Investment under Nonnegative Constraints [J] Operations Research and Management Science, 2001, 10(2):110-113.
[5] Pan Deng, Wu Chongfeng. Using Genetic Algorithm to Directly Search the Efficient Frontier of Portfolio Investment [J] Journal of Systems Engineering, 2002,17(4):364-367

[6] Wang Yanqing, Kang Wansheng, Han Qiheng. Simulation Solution of Portfolio Securities of Probability Criterion Based on Genetic Algorithm [J]. Journal of Management Sciences in China, 2002,5(6):29-34.

[7] Chen Wei. Portfolio Selection Model and Heuristic Algorithm [D] School of Management of Beijing Jiaotong University, 2007,7.

[8] Xia Mengyu, Ye Chunming, Xu Jidong. Using Particle Swarm Optimization to Solve Portfolio Model Containing Transaction Cost [J] Journal of University of Shanghai for Science and Technology, 2008,30(4) : 379-386.

[9] Zhang Hongwen, Chen Zhijiang, Hao Lin. Missile Firepower Optimization Allocation Based on Adaptive Genet ic Algorithm [J] T actical Missile Technology, 2007,(4):28-3O.

[10] Wang Wanliang, Wu Qidi, Song Yi. Solution to Improved Adaptive Genetic Algorithm on Job Shop Scheduling Problem [J] System Engineering Theory and Practice, 2004,(2) : 32-34.

[11] GOURIEROUX C, LAURENT HP,PHAH . Mean-variance hedging and numeraire[J].Mathematical Finance,1998,8(3) : 179200 . 